\title{
Stabilities of isomers of phosphorous on transition metal substrates
}

Yuling Yin ${ }^{a, b}$, Vladislav Gladkikh ${ }^{b}$, Pai Li ${ }^{b}$, Leining Zhang ${ }^{b}$, Qinghong Yuan ${ }^{*}$, Feng Ding $^{*} b, c$

a State Key Laboratory of Precision Spectroscopy, School of Physics and Electronic Science, East China Normal University, 500 Dongchuan Road, Shanghai 200241, People's Republic of China.

${ }^{\mathrm{b}}$ Center for Multidimensional Carbon Materials, Institute for Basic Science (IBS), Ulsan 44919, Republic of Korea.

${ }^{c}$ Department of Materials Science and Engineering, Ulsan National Institute of Science and Technology (UNIST), Ulsan 44919, Republic of Korea.

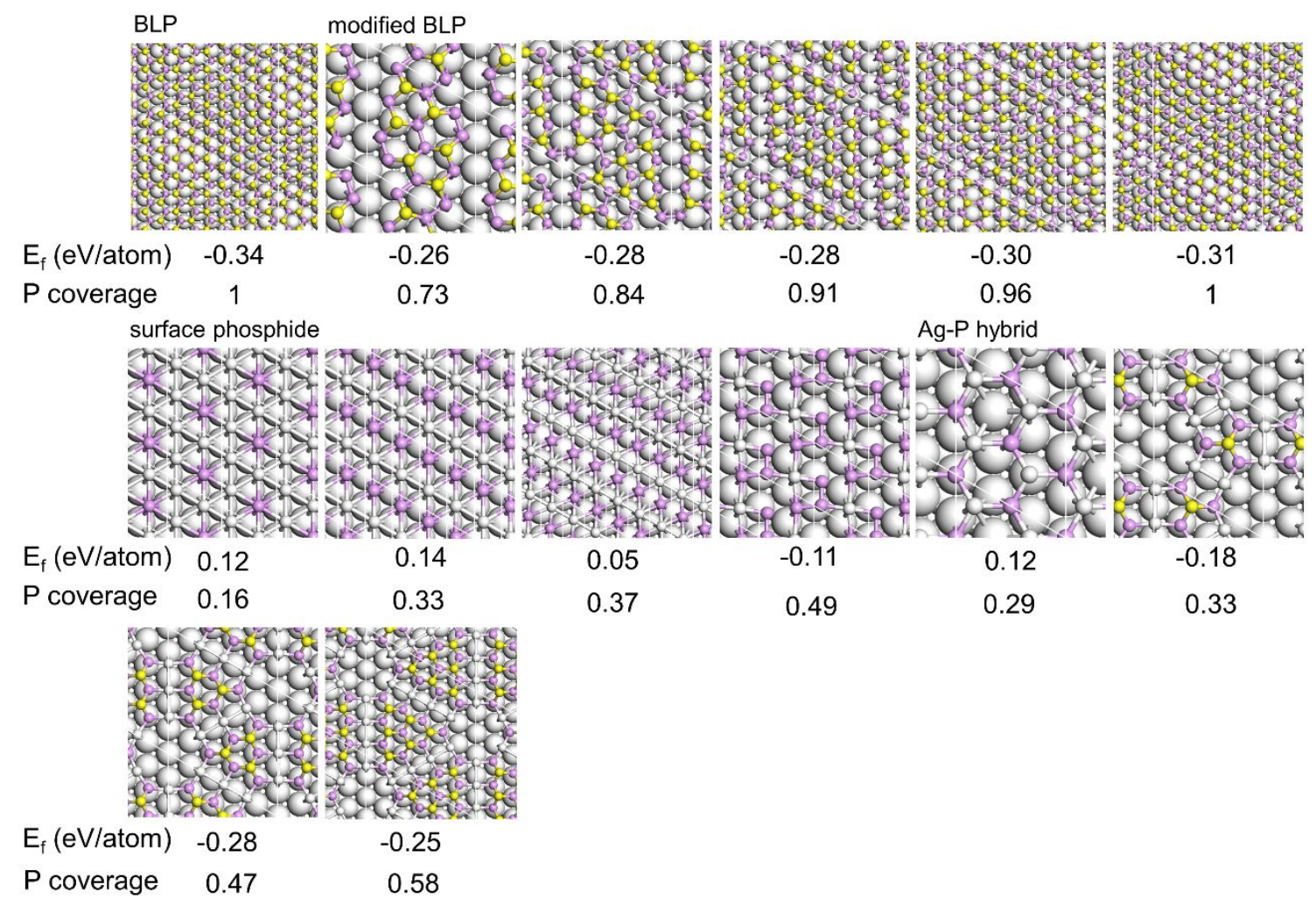


Figure S1. Structures, corresponding formation energies, and P coverages on $\mathrm{Ag}(111)$ surface.

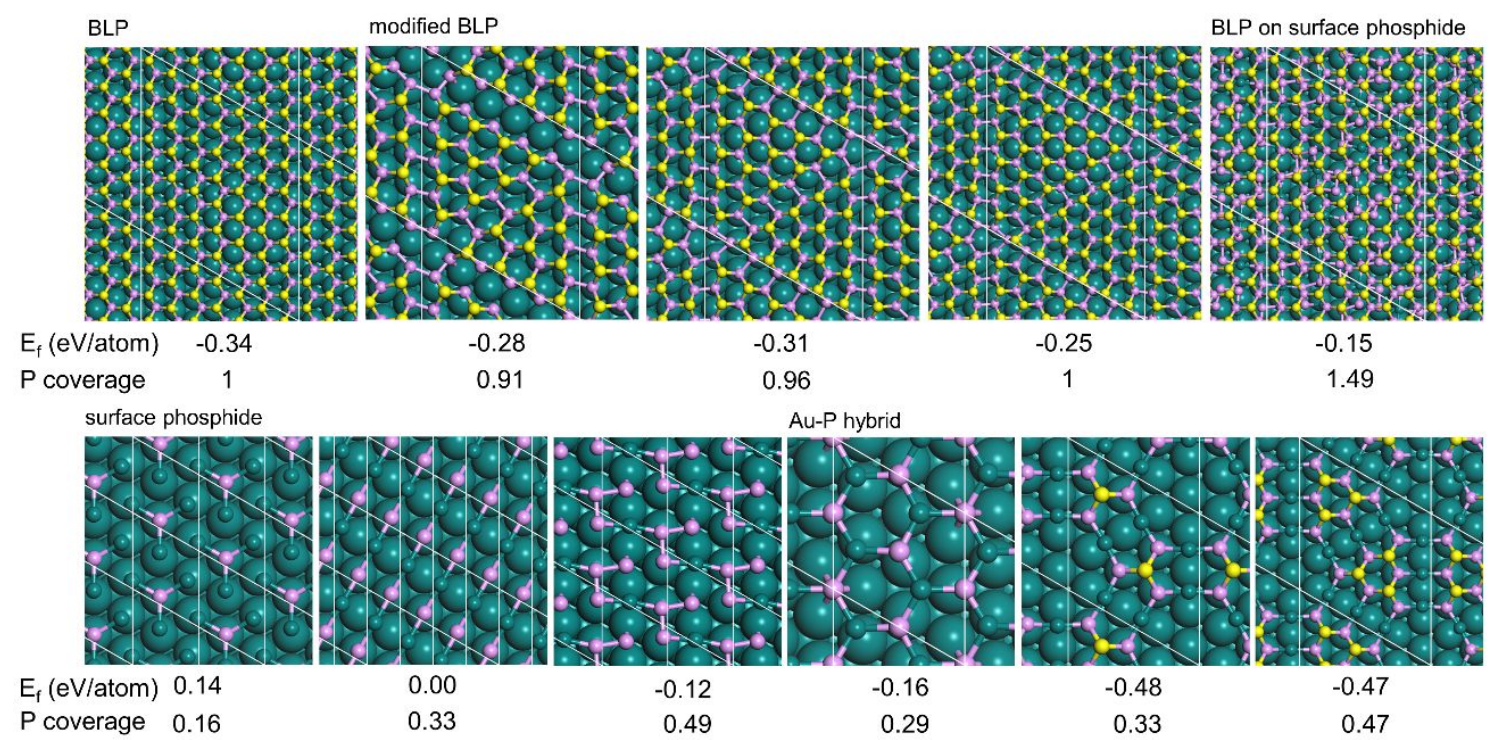

Figure S2. Structures, corresponding formation energies, and P coverages on $\mathrm{Au}(111)$ surface.

BLP

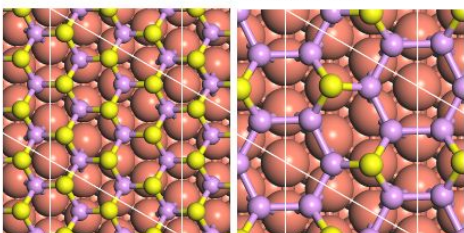

$E_{f}(e V / a t o m)-0.56$

P coverage 1
$-0.44$

0.79

Cu-P hybrid

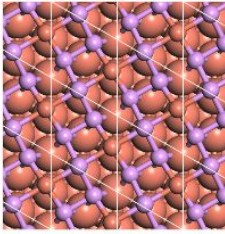

$\mathrm{E}_{\mathrm{f}}(\mathrm{eV} / \mathrm{atom})-0.54$

$P$ coverage $\quad 0.67$

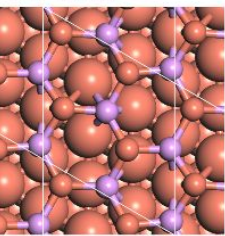

$-0.72$

0.40

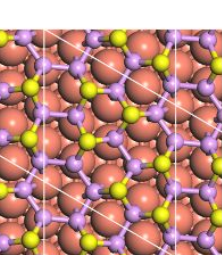

$-0.44$

1

surface phosphide

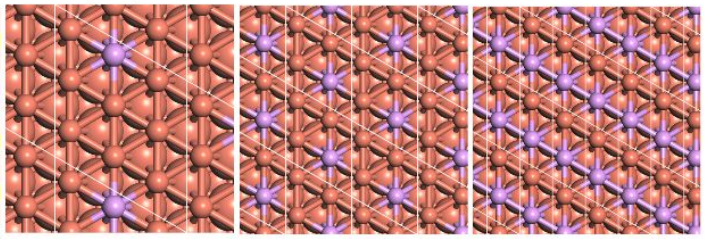

$-1.19$

0.10

0.22

$-0.69$

BLP on surface phosphide

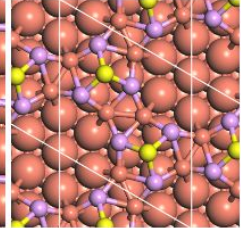

$-0.39$

0.44

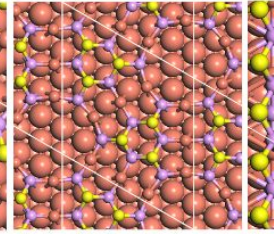

$-0.69$

0.44

1.22

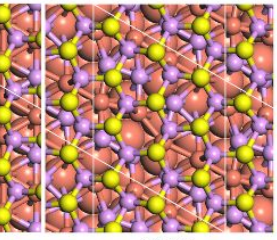

$-0.38$

1.44

Figure S3. Structures, corresponding formation energies, and $\mathrm{P}$ coverages on $\mathrm{Cu}(111)$ surface. 

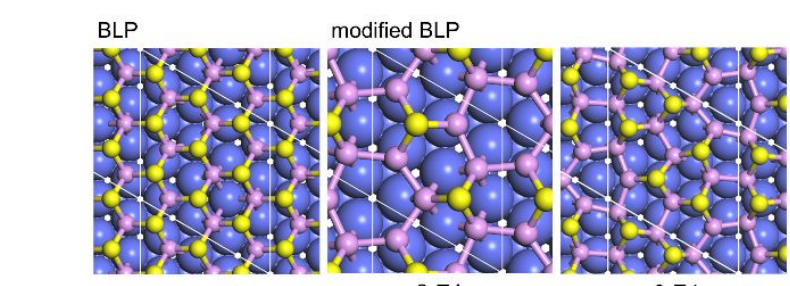

surface phosphide

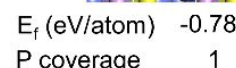

-0.71
0.79

$-0.71$

Co-P hybrid
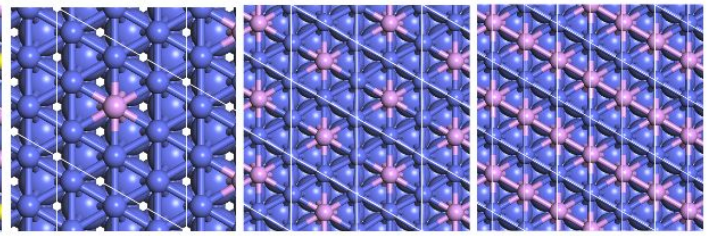

$P$ coverage 1

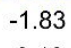

$-1.91$

$-1.50$

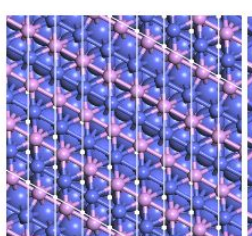

$E_{f}(e V / a t o m) \quad-1.69$

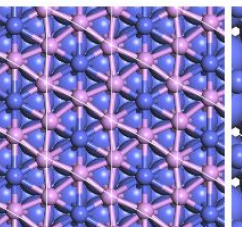

0.10

BLP on surface phosphide

$P$ coverage $\quad 0.51$

$-0.90$

0.67
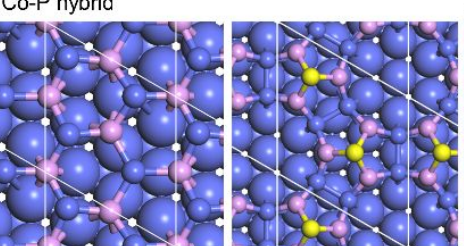

BLP on surface phosphide

0.44

Figure S4. Structures, corresponding formation energies, and P coverages on Co(0001) surface.

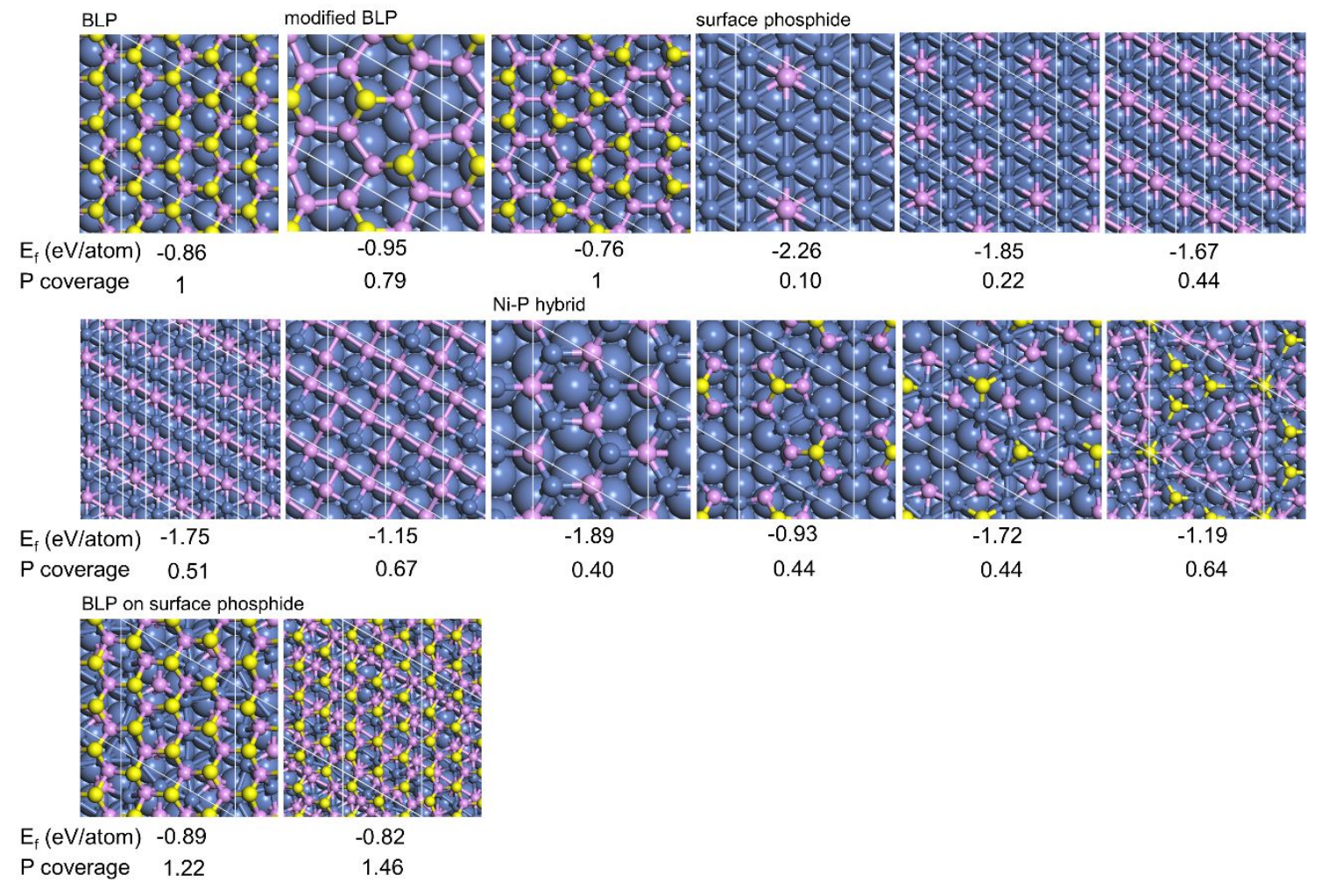

Figure S5. Structures, corresponding formation energies, and P coverages on $\mathrm{Ni}(111)$ surface. 

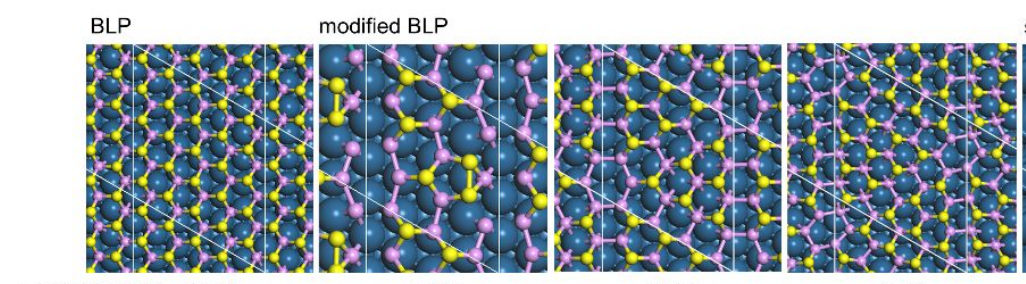

surface phosphide

$\mathrm{E}_{\mathrm{f}}(\mathrm{eV} /$ atom $)-0.79$

-0.82
0.81

-0.77
0.92

$-0.74$

Pt-P hybrid

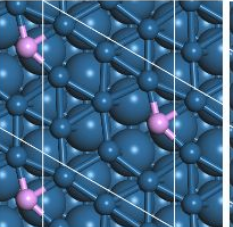

$-0.53$

Pl-P hybrid
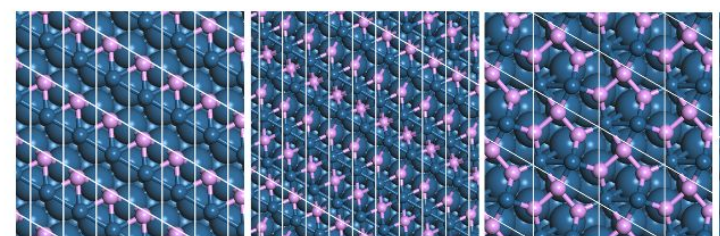

0.08
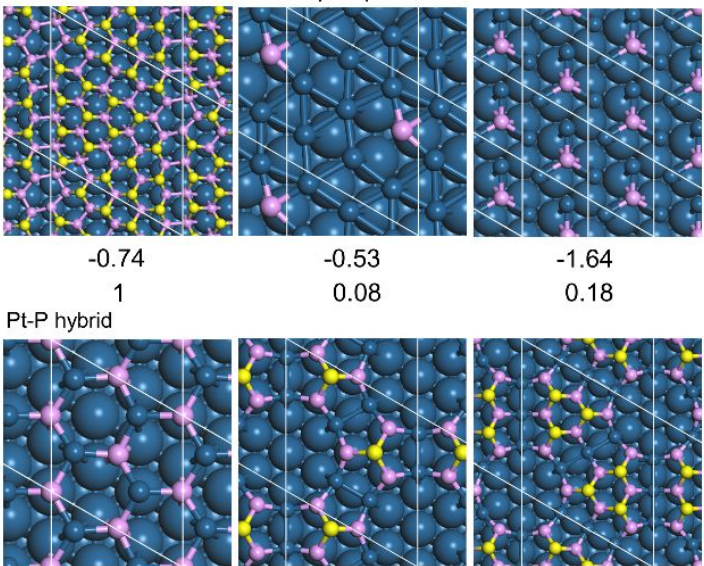

$E_{f}($ eV/atom) -0.94

$P$ coverage 0.36

$-1.37$

0.45

$-1.24$

$-1.40$

$-1.21$

$-1.05$

BLP on surface phosphide

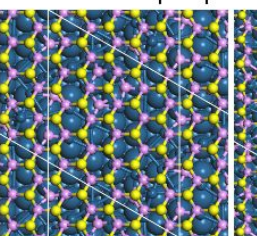

$E_{f}(e V / a t o m)-0.89$

$\mathrm{P}$ coverage 1.18

$-0.72$

1.50

Figure S6. Structures, corresponding formation energies, and P coverages on $\mathrm{Pt}(111)$ surface.

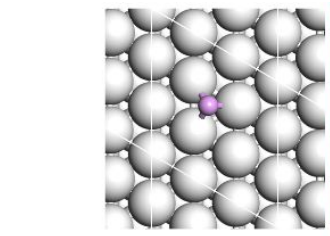

$\mathrm{Ag}(111)$

$\mathrm{E}_{\mathrm{f}}(\mathrm{eV} /$ atom $) \quad 0.48$

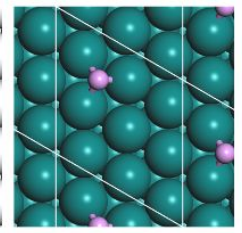

$\mathrm{Au}(111)$

0.20

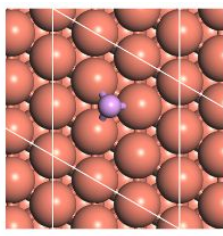

$\mathrm{Cu}(111)$

$-0.48$

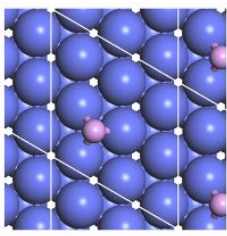

Co(0001)

$-1.39$

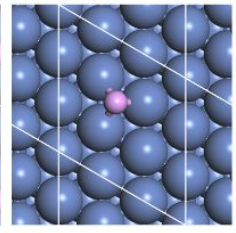

$\mathrm{Ni}(111)$

$-1.65$

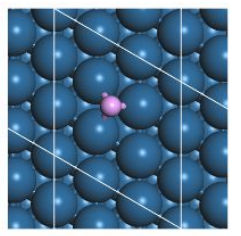

$\mathrm{Pt}(111)$

$-2.06$

Figure S7. The structures and formation energies of a $\mathrm{P}$ atom on $\mathrm{Ag}(111), \mathrm{Au}(111), \mathrm{Cu}(111)$,

$\mathrm{Co}(0001), \mathrm{Ni}(111)$ and $\mathrm{Pt}(111)$ surfaces. 